\title{
RECURSIVE DENSITY TYPES. II
}

\author{
BY \\ H. GONSHOR
}

1. Introduction. The main aim of this paper is to give solutions to Pavlova's three problems and to given an extension of the cancellation law proved in [1]. Results of a more model-theoretic nature will be left to a future paper.

We shall use the notation and definitions of [1]. It turns out that the solution to one of the problems involves sifting in the literature. Although the other two problems interest us more, we include a discussion of the first problem for completeness.

2. Pavlova's three problems. Pavlova [5] asks if there are uniformly hyperimmune sets with r.e. complements. (The term rarefied is used in the translation.) She also asks the same question for strongly hyperimmune sets. (The translation uses the term weakly rarefied.) These questions are essentially answered in the American literature although the motivation and notation is different. An r.e. set is called dense if its complement is strongly hyperimmune. On the other hand, no word for strongly hyperimmune appears in the literature. This is consistent with the historical tradition where the terms simple, creative, and maximal were used before the terms immune, productive, and cohesive.

(a) In [7] it is shown that comaximal sets are strongly hyperimmune. In [3] this is generalized to cohyperhypersimple sets. Finally an adaptation of a proof in [8] gives an r.e. set with uniformily hyperimmune complement. It is enough to vary scheme 1 on p. 466 as follows: change the subscript $e$ in $T_{e}^{n}$ to $U_{e-1}^{n}$ and the subscript $e_{n}$ in $T_{e_{n}}^{n}$ to $U_{e_{n-1}}^{n}$.

(b) As an answer to the next problem we shall show that the lattice of densities $\Delta$ is not implicative. In general a lattice $L$ is said to be implicative if for all $A, B \in L$ there is a maximal $D$ such that $A \cap D \leqq B$. For Boolean algebras it is easily verified that $B \cup A^{\prime}$ serves for the $D$. Implicative lattices are useful for studying the intuitionistic calculus algebraically.

We shall construct a pair of densities $A$ and $B$ such that

$$
\forall D[A \cap D \leqq B \rightarrow(\exists F)(F>D \text { and } A \cap F \leqq B)] .
$$

This will show that there exist pairs $A, B \in \Delta$ such that there is not even a maximal $D$ such that $A \cap D \leqq B$.

Consider an enumeration of the set of all pairs $\left\{\left(h_{i}, h_{j}\right)\right\}$ of strictly increasing recursive functions. For convenience the pairs are indexed by positive integers.

Received by the editors May 17, 1968. 
Define $\alpha$ and $\beta$ inductively as follows : $\alpha(0)=\beta(0)=0, \alpha(1)=\beta(1)=1$. If $n$ enumerates $\left(h_{i}, h_{j}\right)$ then

$$
\begin{aligned}
\alpha(2 n) & =h_{i}[\alpha(2 n-1)+1], \\
\beta(2 n) & =h_{j}[\alpha(2 n)]+1, \\
\alpha(2 n+1) & =\beta(2 n+1)=\beta(2 n)+1 .
\end{aligned}
$$

Now suppose $\gamma$ satisfies $\bar{\alpha} \cap \bar{\gamma} \leqq \bar{\beta}$, i.e., for some strictly increasing recursive $h$, $h\{\max [\alpha(n), \gamma(n)]\} \geqq \beta(n)$ for all $n . h=h_{j}$ for some $j$. Let $i$ be arbitrary and choose $n$ corresponding to $\left(h_{i}, h_{j}\right)$. Then $h_{j} \alpha(2 n)<\beta(2 n)$ by construction. Hence $h_{j} \gamma(2 n) \geqq$ $\beta(2 n)$. Thus $\gamma(2 n)>\alpha(2 n)$.

Now let $\gamma^{\prime}(2 n)=\alpha(2 n-1)+1$ if $n$ corresponds to a pair of the form $\left(h_{i}, h\right)$ and $\gamma^{\prime}(n)=\min [\gamma(n), \beta(n)]$ otherwise. It is easily seen that $\gamma^{\prime}$ is strictly increasing and that $\gamma^{\prime}(n) \leqq \gamma(n)$ for all $n$. For $n$ corresponding to $\left(h_{i}, h\right)$,

$$
\gamma(2 n)>\alpha(2 n)=h_{i}[\alpha(2 n-1)+1]=h_{i} \gamma^{\prime}(2 n) .
$$

Since $i$ is arbitrary it follows that $\bar{\gamma}<\bar{\gamma}^{\prime}$. It remains to show that $\bar{\alpha} \cap \bar{\gamma}^{\prime} \leqq \bar{\beta}$. It is enough to check that $h\left\{\max \left[\alpha(n), \gamma^{\prime}(n)\right]\right\}+1 \geqq \beta(n)$ for all $n$. This is clear for those $n$ such that $\gamma^{\prime}(n)=\min [\gamma(n) \beta(n)]$. If $n$ corresponds to $\left(h_{i}, h\right)$ then

$$
h\left\{\max \left[\alpha(2 n), \gamma^{\prime}(2 n)\right]\right\}+1 \geqq h_{j} \alpha(2 n)+1=\beta(2 n) .
$$

This completes the proof.

This result is expected to play a role in a deeper study of $\Delta$. For example, note that we have an example of a Horn sentence valid in all boolean algebras but not in $\Delta$.

(c) The final question is whether there exists a hyperimmune set which is neither strongly hyperimmune nor equivalent to a cohyperimmune set. This was independently asked by Rice in an unpublished note. This question is basic to the study of subclasses of $\Delta$.

Let $\Delta_{\tau}$ be the class of all densities which do not contain any cohyperimmune sets. It is clear from Theorem 5 in [1] that $\Delta_{s} \subset \Delta_{\tau}$. As an answer to the question we shall show that $\Delta_{\tau}-\Delta_{s}$ is nonempty.

We first prove a lemma.

LEMmA. If $\gamma \in A+B$, then $(\exists \alpha \in A)(\exists \beta \in B)(\alpha \cap \beta=\varnothing$ and $\alpha \cup \beta=\gamma)$.

Proof. Choose $\alpha^{\prime} \in A$ and $\beta^{\prime} \in B$ such that $\alpha^{\prime} \cap \beta^{\prime}=\varnothing$. Then $\gamma \sim \alpha^{\prime} \cup \beta^{\prime}$. Consider the one-one correspondence $\gamma(n) \leftrightarrow\left(\alpha^{\prime} \cup \beta^{\prime}\right)(n)$ where, as usual, $\delta(n)$ ẹnumerates $\delta$ in order of size for any set $\delta$. Now

$$
(\exists \text { recursive } h)(\forall n)\left\{\gamma(n) \leqq h\left[\left(\alpha^{\prime} \cup \beta^{\prime}\right)(n)\right] \text { and }\left(\alpha^{\prime} \cup \beta^{\prime}\right)(n) \leqq h \gamma(n)\right\} .
$$

Restrict the correspondence to $\alpha^{\prime}$ and $\beta^{\prime}$ respectively. This partitions $\gamma$ into two sets $\alpha$ and $\beta$ satisfying the properties required in the lemma. Note, in fact, that the same $h$ works. 
We apply this lemma to obtain an algebraic characterization of $\Delta_{\tau}$.

Corollary. $A \in \Delta_{\tau} \leftrightarrow(\forall B)(B \neq \infty \rightarrow A+B \neq \infty)$.

Proof. If $A \notin \Delta_{\tau}$ choose $\alpha \in A$ with hyperimmune complement $\beta$. If $B=\bar{\beta}$ then $B \neq \infty$ but

$$
A+B=\overline{\alpha \cup \beta}=\infty .
$$

Conversely, suppose that $(\exists B \neq \infty)(A+B=\infty)$. Then apply the lemma to $\gamma=\varepsilon$. We obtain $\alpha \in A$ such that $\alpha^{\prime}=\beta$ is hyperimmune.

It is clear from the corollary that $\Delta_{\tau}$ is an ideal. We now construct a density in $\Delta_{\tau}-\Delta_{s}$.

Let $h_{i}$ be an enumeration of the strictly increasing recursive functions and let $g_{i}=\max _{j \leqq i} h_{j}$. For convenience let $h_{0}(n)=2 n$. Choose $\alpha(0)=g_{0}(0)+1$. We define a sequence $n_{0}, n_{1}, \ldots$ and $\alpha$ inductively as follows. Let $n_{0}=0$. If $n_{i}$ is defined and $\alpha\left(n_{i}\right)=g_{i}\left(n_{i}\right)+1$. Let $n_{i+1}=\alpha\left(n_{i}\right)-n_{i}+1$. Define $\alpha(x)$ as follows:

$$
\begin{array}{ll}
\text { if } \quad n_{i}<x<n_{i+1}, & \alpha(x)=\alpha\left(n_{i}\right)+x-n_{i}, \\
\text { if } x=n_{i+1}, & \alpha(x)=g_{i+1}\left(n_{i+1}\right)+1 .
\end{array}
$$

Note that the choice of $h_{0}$ guarantees that $\alpha\left(n_{i}\right) \geqq 2 n_{i}$ and hence that $n_{i}+1<n_{i+1}$. Thus the construction makes sense. Furthermore

$$
\begin{aligned}
\alpha\left(n_{i+1}-1\right) & =\alpha\left[\alpha\left(n_{i}\right)-n_{i}\right]=\alpha\left[n_{i}+\alpha\left(n_{i}\right)-2 n_{i}\right]=\alpha\left(n_{i}\right)+\left[\alpha\left(n_{i}\right)-2 n_{i}\right] \\
& =2\left[\alpha\left(n_{i}\right)-n_{i}\right]=2\left(n_{i+1}-1\right) .
\end{aligned}
$$

To summarize heuristically, $\alpha$ goes up slowly to let the function $f(n)=2 n$ catch up and then $\alpha$ takes a gigantic leap.

It is immediate that $\bar{\alpha} \notin \Delta_{s}$ since $\alpha(n)=2 n$ infinitely often. To show that $\bar{\alpha} \in \Delta_{\tau}$ we must make $\Delta_{\tau}^{\prime}$ more tractable.

A cohyperimmune set $\beta$ has the following property:

$$
(\forall \text { recursive } h)\left(\forall n_{0}\right)\left(\exists n \geqq n_{0}\right)[\{\beta[h(n)-n]\} \leqq h(n)] .
$$

This follows from the usual characterization of hyperimmune sets by taking complements. For such an $n$ we have the following: $n \leqq x \leqq h(n)-n \rightarrow \beta(x) \leqq 2 x$. This is so because $\beta(x)-x$ is monotonic increasing. Hence $\beta(x)-x \leqq \beta[h(n)-n]$ $-[h(n)-n] \leqq h(n)-[h(n)-n]=n \leqq x$. Thus $\beta(x) \leqq 2 x$. Roughly speaking we have shown that there are "long" runs for which $\beta(x) \leqq 2 x$.

If $\beta^{\prime} \sim \beta$ then ( $\exists$ recursive $g$ ) $(\forall n)\left[\beta^{\prime}(n) \leqq g \beta(n)\right]$. Then $\beta^{\prime}(x) \leqq g(2 x)$ for $n \leqq x$ $\leqq h(n)-n$. Since $h$ is arbitrary we may choose $h(x)=g(2 x)+x$. Then $\beta^{\prime}(x) \leqq g(2 x)$ for $n \leqq x \leqq g(2 n)$. We have shown that

$$
(\exists \text { recursive } h)\left(\forall n_{0}\right)\left(\exists n \geqq n_{0}\right)(\forall x)\left[n \leqq x \leqq h(n) \rightarrow \beta^{\prime}(x) \leqq h(x)\right] .
$$

We must prove that $\alpha$ does not have this property. This is done by contradiction.

Now for $i$ sufficiently high $g_{i} \geqq h$. Choose $n \geqq n_{i}$ such that $n \leqq x \leqq h(n) \rightarrow \alpha(x)$ 
$\leqq h(x)$. No $x$ in the interval can be of the form $n_{j}$ since $\alpha\left(n_{j}\right)>g_{j}\left(n_{j}\right) \geqq h\left(n_{j}\right)$. Hence $\alpha(x)=\alpha(n)+x-n$. If $x=\alpha(n)-n$, then $\alpha(x)=\alpha(n)+\alpha(n)-n-n=2[\alpha(n)-n]=2 x$. (Of course $n \leqq \alpha(n)-n \leqq h(n)$.) Therefore by construction of $\alpha, \alpha(n)-n+1$ is of the form $n_{j}$. This contradicts an earlier statement since $\alpha(n)-n+1 \leqq h(n)$.

We hope to study $\Delta_{\tau}-\Delta_{s}$ in more detail at a future time. In some vague sense the densities in $\Delta_{\tau}-\Delta_{s}$ are analogous to the mesoic sets, i.e., they are the "medium size" densities.

3. The cancellation law for $\Delta_{\tau}$. The main result in this section is:

$$
\left(\forall A \in \Delta_{\tau}\right)(\forall B)(\forall C)(A+B \leqq A+C \rightarrow B \leqq C) .
$$

It is clear that the cancellation law cannot be extended any further since if $A \notin \Delta_{\tau}$ there exists a $B \neq \infty$ such that $A+B=\infty$. Furthermore in view of the result that $\Delta_{\tau}$ properly contains $\Delta_{s}$, this is definitely an extension of the result in [1].

THEOREM. $\left(\forall A \in \Delta_{\tau}\right)(\forall B)(\forall C)(A+B \leqq A+C \rightarrow B \leqq C)$.

Proof. Let $\alpha \in A \in \Delta_{\tau}$ and let $h$ be an arbitrary strictly increasing recursive function. We now define a set $\beta$ as follows:

$$
\beta=\left\{h^{i}(0): \neg(\exists x)\left[x \in \alpha \text { and } h^{i-1}(0)<x \leqq h^{i}(0)\right]\right\}
$$

( $h^{i}$ is the $i$ th iterate of $h$ ). For convenience we use the convention that $h^{0}(n)=n$ and $h^{-1}(n)=+1$, so that when $i=0$ the above interval is simply $\{0\}$. Now $\alpha \cup \beta$ has at least one element in each interval $h^{i-1}(0)<x \leqq h^{i}(0)$. Hence $(\alpha \cup \beta)(n) \leqq h^{n}(0)$. Thus $\alpha \cup \beta$ is not hyperimmune. Since $\alpha \in \Delta_{\imath}, \beta$ is not hyperimmune. Therefore ( $\exists$ strictly increasing recursive $g)(\forall n)[\beta(n) \leqq g(n)]$.

Let $n$ be arbitrary. Then $(\exists i \leqq n)\left[h^{i-1}(0)<n \leqq h^{i}(0)\right]$. Now for all $n \beta(n) \leqq g(n)$ $\leqq h^{g(n)}(0)$ since $h$ is strictly increasing. Hence $\beta(n+1) \leqq h^{g(n+1)}(0)$. Thus $\beta$ has at least $n+2$ elements not greater than $h^{g(n+1)}(0)$. Since $i \leqq n$, at least one of these elements is greater than $h^{i}(0)$. Thus $(\exists j)\{[i<j \leqq g(n+1)]\}$ and $h^{j}(0) \in \beta$. By construction of $\beta$, it follows that $\alpha$ has no elements $x$ satisfying $h^{j-1}(0)<x \leqq h^{j}(0)$. Thus we finally obtained a condition that does not involve $\beta$, namely the following: $(\forall h)(\exists g)(\forall n)(\exists j)\left[n \leqq h^{J-1}(0), j \leqq g(n+1)\right.$, and $\alpha$ has no elements $x$ satisfying $\left.h^{j-1}(0)<x \leqq h^{j}(0)\right]$. Roughly speaking we obtained the existence of "large gaps" in $\alpha$.

Now suppose $A+B \leqq A+C$. Let $\alpha \in A, \beta \in B, \gamma \in C$ such that $\alpha \cap \beta=\alpha \cap \gamma=\varnothing$. Then $\alpha \cup \beta \in A+B$ and $\alpha \cup \gamma \in A+C$.

Then $(\exists h)(\forall x)\{n[\alpha \cup \gamma, h(x)] \geqq n(\alpha \cup \beta, x)\}$. Choose $g$ as above corresponding to $h$. Let $n$ be arbitrary and then choose $j$ as above. Then

$$
n\left[\alpha \cup \gamma, h^{j}(0)\right] \geqq n\left[\alpha \cup \beta, h^{j-1}(0)\right] .
$$

Now finally the above condition is just what is needed to get rid of $\alpha$ to obtain $n\left[\gamma, h^{j}(0)\right] \geqq n\left[\beta, h^{j-1}(0)\right]$. Hence a fortiori $n\left[\gamma, h^{g(n+1)}(0)\right] \geqq n[\beta, n]$. Since $h^{g(n+1)}(0)$ is a recursive function of $n$ this shows that $B \leqq C$. 
Corollary. $\left(\forall A \in \Delta_{\tau}\right)(A+B=A+C \rightarrow B+C)$.

This result diminishes the importance of $\Delta_{s}$ and shifts the emphasis to $\Delta_{\tau}$.

4. Final remarks. The density theorem (Theorem 3) in [1] can be strengthened by, ironically enough, using an easier construction. We let $h_{i}$ be an enumeration of the strictly increasing recursive functions and $g_{i}=\max _{J \leqq i} h_{J}$. The set of $g$ 's then have the property that unboundedness by any infinite subset already implies recursive unboundedness. We now run through the $g$ 's only once.

We now vary the construction of $\gamma_{c}$ as follows: Whenever $c_{l(i)}$ appears change $c_{l(i)}$ to $c_{i}$. Again $(\forall i)\left(c_{i} \geqq d_{i} \rightarrow \gamma_{c} \geqq \gamma_{d}\right)$. However, the map $c \rightarrow \gamma_{c}$ is no longer one-one. But in view of the above property of the $g$ 's, $\gamma_{c}=\gamma_{d} \leftrightarrow c_{i}=d_{i}$ for all but finitely many $i$. It is easily seen that we get a sublattice isomorphic to the Boolean algebra of subsets of a countable set modulo finite sets. This appears to be weaker than what was obtained in [1]. However, according to [2] this Boolean algebra is homogeneous universal. Hence, assuming the continuum hypothesis every Boolean algebra of cardinality $\leqq c$ is embeddable into the class of densities $H$ satisfying $A<H<B$. It follows that any distributive lattice of cardinality $\leqq c$ can be so embedded. Incidentally, by a standard trick, the Boolean algebra of subsets of a countable set can be explicitly embedded in this algebra. Thus Theorem 3 in [1] follows even without using the continuum hypothesis.

Finally, Peter Aczel recently developed a theory analogous to that of recursive combinatorial functions and obtained results analogous to those of Nerode's for isols. The theory for densities turns out to be simpler. After further study, these results will be discussed in future papers.

Added in Proof. It was recently noticed that all comaximal sets are uniformly hyperimmune. Thus the adaptation of the proof in [8] is superfluous.

\section{BIBLIOGRAPHY}

1. H. Gonshor and G. Rice, Recursive density types. I, Trans. Amer. Math. Soc.

2. J. Keisler, Universal homogeneous Boolean algebras, Michigan Math. J. 13 (1966), 129-132.

3. D. Martin, A theorem on hyperhypersimple sets, J. Symbolic Logic 28 (1963), 273-278.

4. Yu. T. Medvedev, On nonisomorphic recursively enumerable sets, Dokl. Akad. Nauk SSSR 102 (1955), 211-214.

5. E. A. Pavlova, Denseness of hyperimmune sets, Dokl. Akad. Nauk SSSR 139 (1961), 814-817 = Soviet Math. Dokl. 2 (1961), 1017-1020.

6. - The lattice of denseness of sets of natural numbers, Izv. Moldavsk Fil. Akad. Nauk SSSR 1960, no. 10(76), 31-38.

7. S. Tennenbaum, Degree of unsolvability and the rate of growth of functions, Proc. Sympos. Math. Theory of Automata, New York, 1962, pp. 71-73.

8. C. E. M. Yates, Three theorems on the degree of recursively enumerable sets, Duke J. Math. 32 (1965), 461-468.

Rutgers, The State University, New Brunswick, New Jersey 